\title{
Combining Ability for Yield and its Attributes in Response to Arsenic Absorption in Maize (Zea mays L.)
}

\author{
Girish Nandan, Shyam Sundar Mandal*, \\ Bishnu Deo Prasad, Urusha Zareen and Jyoti Rani
}

Department of Plant Breeding and Genetics, BAC, Bihar Agricultural University, Sabour, Bhagalpur, India-813210

*Corresponding author

\section{A B S T R A C T}

\section{Keywords}

Combining ability,

Heterosis, GCA,

SCA, Maize (Zea mays L.), Nature of gene action

Article Info

Accepted:

18 May 2020

Available Online:

10 June 2020
Combining ability was studied in a $4 \times 4$ half diallel cross in maize for grain yield and yield contributing characters under arsenic treatment. Half diallel analysis is an efficient method for the study of combining ability and the gene action of the characters under study. Combining ability (GCA and SCA) refers to the ability of a genotype to transmit superior performance to its crosses. Its estimation are important genetic attributes for maize breeders in anticipating improvement in productivity via hybridization and selection. An estimate of combining ability analysis is one of the powerful tools for the identification of best combiners that may be used in crosses either to exploit heterosis or to accumulate productive genes which can further be utilized for genetic improvement for yield.

\section{Introduction}

Maize (Zea mays L.; $2 n=20$ ) is the third most important cereal crop after paddy and wheat in India. Globally, it continues to expand rapidly at an average rate of $20 \%$ per year (CIMMYT, 2008). It has assumed greater significance owing to its demand as food for human; feed for livestock and poultry and as raw material for industries. The total production of maize in India has taken a giant leap from merely 1.73 million tonnes in the year $1950-1951$ to 21.81 million tonnes in the year 2015-2016, which is almost 12.6 times that of 1950-1951. It covered an area of $8.7 \mathrm{~m}$ ha with mean yield of $2.51 \mathrm{t} \mathrm{ha}^{-1}$. (Directorate of Economics \& Statistics, Ministry of Agriculture \& Farmer Welfare, India, 20152016). Bihar is one of the leading maize producing states after Andhra Pradesh \& Karnataka in India with the annual production of 2.4 million tonnes over an area of 7.02 lakh hectare. The average yield of maize in Bihar accounting for $5.34 \mathrm{t} \mathrm{ha}^{-1}$, was higher than the country's average yield i.e. $2.51 \mathrm{t} \mathrm{ha}^{-}$ ${ }^{1}$.(Directorate of Economics \& Statistics, 
Ministry of Agriculture \& Farmer Welfare, India, 2016-2017).

\section{Combining ability and heterosis for yield and its attributes}

Diallel mating designs provide a very simple and convenient method for the estimation of genetic parameters. Among various diallel forms, the half diallel techniques have certain advantages over others, giving maximum information about genetic architecture of a trait, parents and allelic frequency (Kearsey, 1965). Half diallel analysis is an efficient method for the study of combining ability and the gene action of the characters under study. Combining ability refers to the ability of a genotype to transmit superior performance to its crosses. Its estimation are important genetic attributes for maize breeders in anticipating improvement in productivity via hybridization and selection. An estimate of combining ability analysis is one of the powerful tools for the identification of best combiners that may be used in crosses either to exploit heterosis or to accumulate productive genes which can further be utilized for genetic improvement for yield.

\section{Effect of arsenic on maize}

Arsenic (As) is a highly toxic metalloid present ubiquitously in nature that represents a potential environment threat to human, animal and plant health. High arsenic levels in farming zones can affect plant development and decrease agricultural production and could add substantial amounts of arsenic to the diet intake through agricultural product consumption, thus posing additional risk to human health (Yu et al., 2009). Arsenate $[\mathrm{As}(\mathrm{V})]$ and phosphate are chemically similar, and are taken up in to plant roots by the same mechanism (Sharples et al., 2000), therefore, high arsenic content may inhibit phosphorus uptake by the plant. In maize high arsenic concentration (50 to $100 \mathrm{mg} / \mathrm{kg}$ ) decrease the yield by reduced ear length kernel no. per row and kernel weight and also decreases grain nutritional quality by reducing the contents of crude fat, crude starch and crude protein (Xiao-ke et al., 2012). Crude fat, crude protein and crude starch are three main factors of nutrition quality of maize grain (Dong, 2006). At low concentrations of arsenic in soil (12.5 to $25 \mathrm{mg} / \mathrm{kg}$ ), the level of crude protein and crude starch increased significantly and crude fat increased slightly, but at high level of arsenic in soil (50 to $100 \mathrm{mg} / \mathrm{kg})$ it decreases significantly.

Thus, understanding, distribution and speciation of arsenic within the edible portions of maize is essential for the estimation of risk and the establishment of effective strategies to gain high yield under arsenic contaminated area is need of hour. Therefore, the present investigation with $4 \times 4$ half diallel cross was undertaken for isolating superior inbred lines and thereby to identify better combining parents to obtain suitable hybrids.

\section{Materials and Methods}

Four diverse maize inbred lines viz. P1 (ZL14501), P2 (VL-1010762), P3 (VL-145312), P4 (VL-1055) were crossed in a diallel fashion excluding the reciprocals during the rabi season in 2017-18. The resulting six F1's and their four parents were evaluated along with two checks (P-3355 and DHM-117) during the Rabi 2018-19. The resulting 6 F1's and their 4 parents were evaluated along with two checks (P-3355 and DHM-117) in completely randomized design at the Maize section of Bihar Agricultural University, Sabour, Bhagalpur, Bihar. A pot experiment was conducted to investigate the effect of different Arsenic (As) levels on maize (Zea mays L.). $6 \mathrm{~kg}$ soil per pot was taken with control and two treatments $(50 \mathrm{mg}$ and 100 $\mathrm{mg}$ arsenic per $\mathrm{kg}$ soil) of arsenic in three replications. The seeds of inbred, their crosses 
and checks were sown by hand dibbling with two seeds per pot. After one week of germination, one seedling from each pot was uprooted to maintain optimum plant population. The recommended doses of fertilizers, weeding, irrigation and other recommended cultural practices were followed to raise the healthy crop. Data were recorded on each pot's plant for plant height $(\mathrm{cm})$, ear height $(\mathrm{cm})$, days to tasseling and silking, ASI, days to maturity, yield per plant,1000 kernel weight, harvest index and shelling percentage. General combining ability (GCA) and specific combining ability (SCA) were estimated following Model I, Method II of Griffing (1956). The mean squares for GCA and SCA were tested against error variance desired using the mean data of all the single cross hybrids and check variety, was estimated and tested according to Singh and Singh (1994).

\section{Results and Discussion}

\section{ANOVA}

Analysis of variance under control conditions for fourteen characters under study

The values of mean sum of square (MSS) of replication for all the traits were founded nonsignificant. This indicated that there is no variation present within the genotype which is planted in three replications. Whereas the values of MSS for treatment were significant for all the traits which indicated that there is considerable amount of variation present among the genotype for all the characters except plant height and ear height. The values of MSS of parents for all the traits were significant. This indicates that there is considerable amount of variation were present among the parents. The value of MSS of hybrids (which developed from half diallele matting design) were significant for all the traits, indicated that all the hybrids were different for all the studied characters (Table.1).

\section{Analysis of variance under treatment 1 (50 $\mathrm{mg} / \mathrm{kg}$ arsenic in soil) for fourteen characters under study}

The value of ANOVA for all 14 studied characters indicated that there is no variation present in the plants of same genotype which revealed from non-significant value of MSS for all the characters. The MSS of treatment for all the characters were significant, which indicates that the variation present among the genotypes in controlled conditions were also present in treatment 1 conditions and also same for parents and hybrids (Table.1).

Analysis of variance under treatment 2 (100 $\mathrm{mg} / \mathrm{kg}$ arsenic in soil) for fourteen characters under study

In treatment 2 there is no variations found among the plants of the same genotypes due to non-significant value of MSS whereas the MSS value for treatment, parents and hybrids were significant for all traits shows that there is variation present among them.

Estimation of general combining ability effects of parents under control and both treatments

\section{Days to $50 \%$ anthesis}

The range for the estimates of GCA effect for days to $50 \%$ anthesis under control varied from -2.27 (P3) to 1.33 (P1). Two parents, P1 and P4 (0.66) showed highly positive significant and one parent P3 showed highly significant negative GCA effect in desirable direction for $50 \%$ anthesis. Under treatment 1 and treatment 2, parent P3 also showed highly significant negative GCA effect in desirable direction for $50 \%$ anthesis. So parent P3 was identified as good general combiners for days to $50 \%$ anthesis under all conditions (Table.2). 


\section{Days to $50 \%$ silking}

The estimates of GCA effect for days to 50\% silking under control and treatment 1 showed that parent P3 showed highly significant negative GCA effect in desirable direction for $50 \%$ silking. While under treatment 2 , parent P3(-1.11) also showed highly significant negative and parent P2 showed significant negative for $50 \%$ silking. So parent P3 was identified as good general combiners for days to $50 \%$ silking under control and treatment 1 conditions whereas under treatment 2 parents, $\mathrm{P} 2$ and P3 were identified as good general combiners for days to $50 \%$ silking.

\section{Anthesis- Silking interval (ASI)}

The estimates of GCA effect for ASI under control conditions and under treatment 1 parent $\mathrm{P}_{1} \quad(-0.50$ equal for both) showed highly significant negative GCA effect in desirable direction for ASI. While under treatment 2, parent P2 (-1.05) also showed highly significant negative. So parent P1 was identified as good general combiners for ASI under control and treatment 1 conditions whereas under treatment 2, parent P2 was identified as good general combiners ASI.

\section{Days to $75 \%$ brown husk}

The estimates of GCA effect for days to $75 \%$ brown husk under control showed that parent P3 (-2.87) showed highly significant negative GCA effect and also for treatment 1, parent P3 (-0.64) showed highly significant negative GCA effect in desirable direction for $75 \%$ brown husk. While under treatment 2, parent P3 (-2.0) showed highly significant negative. So parent P3 was identified as good general combiners for days to $75 \%$ brown husk.

\section{Plant height}

GCA effect for plant height under control for parent P4 (-10.64) was significant negative, under treatment 1 parent $\mathrm{P} 4(-10.60)$ and under treatment 2 parent $\mathrm{P} 4(-10.40)$ were highly significant negative GCA effect in desirable direction showed that parent P4 was identified as good general combiners for plant height in all conditions.

\section{Ear height}

GCA effect for ear height under control for parent P4 (-5.99) was significant negative, under treatment 1 no any parent were significant and under treatment 2 parent P4 (5.79) were significant negative GCA effect in desirable direction showed that parent P4 was identified as good general combiners for ear height in both control and treatment 2 conditions.

\section{Cob length}

GCA effect for cob length under control for parent P2 (1.12) and P3 (1.13) were found to be highly significant positive, under treatment 1, parent P2 (1.12) and P3 (1.08) and under treatment 2, parent P2 (1.15) and parent P3 (1.04) were also highly significant positive GCA effect in desirable direction showed that parent P2 and P3 were identified as good general combiners for cob length in all conditions.

\section{Cob diameter}

GCA effect for cob diameter under control for parent P2 (0.22), under treatment 1 for parent P2 (0.24) and under treatment 2 for parent P2 $(0.23)$ were highly significant positive GCA effect in desirable direction showed that parent P2 was identified as good general combiners for cob diameter in all conditions.

\section{Number of kernal's rows per cob}

GCA effect for number of kernal's rows per cob under control for parent P3 (0.44) was significant positive while under both 
treatment no any parents were significant. Therefore under control positive GCA effect in desirable direction of parent P3 was identified as good general combiners for number of kernal's rows per cob.

\section{Number of kernals per row}

GCA effect for Number of kernals per row under control for parent P2 (3.08) and P3 (1.47) were found to be highly significant positive, under treatment 1 parent P2 (3.0) was highly significant positive and P3 (1.31) was significant positive GCA effect whereas under treatment 2 parent P2 (2.93) and parent P3 (1.32) were also significant positive GCA effect in desirable direction showed that parent P2 and P3 were identified as good general combiners for number of kernels per row in all conditions.

\section{Test weight}

GCA effect for test weight under control for parent P2 (19.50 gm) and P3 (13.06) were found to be highly significant positive and also under treatment 1 parent P2 (20.37) and P3 (12.17) were highly significant positive GCA effect whereas under treatment 2 parent P2 (5.05) and parent P3 (7.61) were also highly significant positive GCA effect in desirable direction showed that parent $\mathrm{P} 2$ and P3 were identified as good general combiners for test weight under all conditions.

\section{Harvest index}

GCA effect for harvest index under control for parent P2 (3.63\%) and P3 (2.26\%) were found to be highly significant positive and also under treatment 1 parent P2 $(3.67 \%)$ and P3 $(2.09 \%)$ were highly significant positive GCA effect whereas under treatment 2 parent P2 $(3.68 \%)$ and parent P3 $(2.10 \%)$ were also highly significant positive GCA effect in desirable direction showed that parent P2 and
P3 were identified as good general combiners for harvest index under all conditions (Table.3).

\section{Shelling percentage}

GCA effect for shelling percentage under control for parent P2 (7.14\%) and P3 (7.72\%) were found to be highly significant positive and also under treatment 1 parent P2 (7.20\%) and P3 (7.38\%) were highly significant positive GCA effect whereas under treatment 2 parent P2 $(7.13 \%)$ and parent P3 $(7.13 \%)$ were also highly significant positive GCA effect in desirable direction showed that parent P2 and P3 were identified as good general combiners for shelling percentage under all conditions (Table.4).

\section{Grain yield per plant}

GCA effect for grain yield under control for parent $\mathrm{P}_{1}$ (2.93 gm), P2 (13.56 gm) and P3 $(7.72 \mathrm{gm})$ were found to be highly significant positive and under treatment 1 and 2 parent P1, P2 and P3 were also highly significant positive GCA effect in desirable direction showed that parent P2 and P3 were identified as good general combiners for grain yield under all conditions.

Estimation of specific combining ability (SCA) effects of crosses under control and both treatments

\section{Days to $50 \%$ tasseling}

SCA effect for days to $50 \%$ tasseling under control for crosses P1 X P2 (-1.67) and P1 X P4 (-1.73) were significantly negative in desirable direction. While for other crosses gca effect were positive. Under treatment 1, P1 X P3 (1.68) and P2 X P3 (2.18) were significantly positive whereas under treatment 2, crosses P1 X P4 (-1.70) was significant negative in desirable direction (Table.5). 


\section{Days to $50 \%$ silking}

SCA effect for days to $50 \%$ silking under control for crosses P1 X P4 (-2.0) was highly significantly negative in desirable direction. While for other crosses P2 X P3 (3.0) gca effect were highly significantly positive. Under treatment 1 , no any crosses were significant whereas under treatment 2, crosses P1 X P4 (-1.80) was significant negative in desirable direction.

\section{Anthesis-silking interval (ASI)}

For ASI no any crosses were show siginificant gca effect under control and treatment 1.Under treatment 2, crosses P2 X P3 (-2.87) and P3 X P4 (-2.26) were significantly negative in desirable direction.

\section{Days to $75 \%$ brown husk}

SCA effect for days to $75 \%$ brown husk under treatment 1 for crosses P1 X P4 (-3.19) and P2 X P4 (-4.28) were significantly negative in desirable direction while under treatment 2 for cross P2 X P4 (-3.82) was significant negative in desirable direction whereas no any crosses were significant under control for days to $75 \%$ brown husk.

\section{Plant height}

SCA effect for plant height under treatment 1 for crosses P3 X P4 (-21.32) was significantly negative in desirable direction while under treatment 2 for cross P3 X P4 (-20.96) was also significantly negative in desirable direction whereas no any crosses were significant under control for plant height

\section{Ear height}

SCA effect for ear height under treatment 1 for crosses P3 X P4 (-16.7) was significantly negative in desirable direction while under treatment 2 for cross P3 X P4 (-17.1) was also significantly negative in desirable direction whereas no any crosses were significant under control for plant height.

\section{Cob length}

SCA effect for cob length under control for crosses P1 X P2 (1.61) and P1 X P3 (1.73) were highly significant positive in desirable direction. Under treatment 1 , crosses P1 X P2 (1.72) and P1 X P3 (1.64) were highly significant positive whereas under treatment 2, crosses P1 X P2 (1.67) and P1 X P3 (1.83) were also highly significant positive in desirable direction.

\section{Cob diameter}

SCA effect for cob diameter under control for cross P1 X P3 (0.51) was significantly positive in desirable direction. Under treatment 1, P1 X P3 (1.64) cross was significantly positive whereas under treatment 2, cross P1 X P3 (0.51) was also highly significant positive in desirable direction.

\section{Number of kernel's rows per cob}

SCA effect for number of kernel's rows per cob under treatment 1 for cross P1 X P2 (1.56) was significantly positive in desirable direction while under treatment 2 for cross P2 X P3 (1.71) was also significantly positive in desirable direction whereas no any crosses were significant under control for number of kernel's rows per cob.

\section{Number of kernels per row}

SCA effect for number of kernels per row under control for cross P1 X P2 (5.80) was highly significant positive in desirable direction. Under treatment 1, P1XP2(5.54) cross was highly significant positive and cross $\mathrm{P} 1 \mathrm{XP} 3(3.17)$ was significantly positive, where as under treatment 2, cross P1 X P2 (5.40) was highly significant positive and P1 X P3 (3.04) was also significantly positive in desirable direction for number of kernels per row. 
Table.1 ANOVA for 14 traits of maize under control, Treatment 1(T1) and treatment 2 (T2) condition

\begin{tabular}{|c|c|c|c|c|c|c|c|c|c|c|c|c|c|c|c|c|}
\hline \multirow{8}{*}{$\begin{array}{l}\mathbf{C} \\
\mathbf{O} \\
\mathbf{N} \\
\mathbf{T} \\
\mathbf{R} \\
\mathbf{O} \\
\mathbf{L}\end{array}$} & $\begin{array}{l}\text { Source of } \\
\text { variation }\end{array}$ & $\begin{array}{l}\text { D } \\
\text { F }\end{array}$ & $\begin{array}{l}\text { DOF } \\
\mathbf{5 0 \%} \mathrm{T} \\
\text { (Days) }\end{array}$ & $\begin{array}{l}\text { DOF } \\
50 \% \quad S \\
\text { (Days) }\end{array}$ & $\begin{array}{l}\text { ASI } \\
\text { (Days) }\end{array}$ & $\begin{array}{l}\text { DOF } \\
75 \% \text { B } \\
\text { (Days) }\end{array}$ & $\begin{array}{l}\mathbf{P H} \\
(\mathbf{c m})\end{array}$ & $\begin{array}{l}\mathbf{E H} \\
(\mathbf{c m})\end{array}$ & $\begin{array}{l}\mathbf{C L} \\
(\mathbf{c m})\end{array}$ & $\begin{array}{l}\text { CD } \\
(\mathbf{c m})\end{array}$ & $\begin{array}{l}\text { NKRP } \\
\text { C }\end{array}$ & NKPR & $\begin{array}{l}1000 \\
\text { GW(gm) }\end{array}$ & HI (\%) & SP (\%) & $\begin{array}{l}\text { GY/per } \\
\text { plant } \\
\text { (gm) }\end{array}$ \\
\hline & Replicates & 2 & 0.43 & 0.433 & 2.33 & 6.63 & 315.00 & 108.90 & 0.14 & 0.20 & 0.40 & 33.10 & 322.13 & 5.58 & 4.77 & 9.56 \\
\hline & Treatments & 9 & $27.83 * *$ & $18.96 * *$ & $1.83 * *$ & $40.10 * *$ & 830.83 & 439.00 & $17.70 * *$ & $0.56 * *$ & $2.66 *$ & $94.55 * *$ & $3751.65 * *$ & $118.18 * *$ & $650.16 * *$ & $2129.40 * *$ \\
\hline & Parents & 3 & $78.75 * *$ & $50.97 * *$ & $4.556 * *$ & $110.08 * *$ & 1114.41 & 314.41 & $30.42 * *$ & $0.80 * *$ & 0.33 & $121.41^{*}$ & $5623.66 * *$ & $187.16^{* *}$ & $1031.12 * *$ & $3626.88 * *$ \\
\hline & Hybrids & 5 & 1.92 & 2.18 & 0.53 & 2.53 & 826.37 & $589.66 *$ & $13.57 * *$ & $0.523 * *$ & $4.48 * *$ & $96.13 * *$ & $3375.02 * *$ & $100.31 * *$ & $550.57 * *$ & $1446.65^{* *}$ \\
\hline & $\begin{array}{l}\text { Parent } \\
\text { Vs.Hybrids }\end{array}$ & 1 & 4.67 & $6.80 *$ & 0.20 & 18.05 & 2.56 & 59.51 & 0.15 & 0.03 & 0.55 & 6.00 & 18.68 & 0.10 & 5.23 & $1050.67 * *$ \\
\hline & Error & $\begin{array}{l}1 \\
8\end{array}$ & 1.24 & 1.39 & 0.37 & 6.15 & 357.42 & 194.16 & 0.14 & 0.17 & 0.84 & 7.36 & 201.02 & 2.44 & 5.24 & 104.59 \\
\hline & Total & $\begin{array}{l}2 \\
9\end{array}$ & 9.44 & 6.78 & 0.80 & 16.72 & 501.44 & 264.28 & 5.60 & 0.25 & 1.39 & 36.21 & 1311.29 & 38.59 & 205.35 & 726.38 \\
\hline \multirow{7}{*}{$\begin{array}{l}\mathbf{T} \\
\mathbf{1}\end{array}$} & Replicates & 2 & 0.43 & $7.23 *$ & 2.13 & 5.31 & 348.10 & 104.67 & 0.07 & 0.03 & 2.00 & $23.45^{*}$ & 75.70 & $13.06^{*}$ & 5.16 & 17.30 \\
\hline & Treatments & 9 & $19.85^{* *}$ & $9.05 * *$ & $12.05 * *$ & $44.82 * *$ & $713.36 *$ & 349.16 & $17.06^{* *}$ & $0.54 * *$ & $3.95 *$ & $83.01 * *$ & $1202.60 * *$ & $116.69 * *$ & $611.19 * *$ & $2247.40 * *$ \\
\hline & Parents & 3 & 52.30 & $18.30 * *$ & $18.30 * *$ & $90.25 * *$ & 954.00 & $268.87^{*}$ & $28.73 * *$ & $0.77 * *$ & 1.22 & $106.54 *$ & $238.52 * *$ & $185.71 * *$ & $974.83 * *$ & $4232.35 * *$ \\
\hline & Hybrids & 5 & 1.95 & 8.05 & $8.05^{*}$ & $21.08 *$ & 709.70 & $460.06^{*}$ & $13.42 * *$ & $0.51 * *$ & $6.22 * *$ & $84.66 * *$ & $2009.78 * *$ & $98.62 * *$ & $514.00 * *$ & $1374.83 * *$ \\
\hline & $\begin{array}{l}\text { Parent } \\
\text { Vs.Hybrids }\end{array}$ & 1 & $12.22 * *$ & $13.39 *$ & $13.39 *$ & $27.48^{*}$ & 9.75 & 35.56 & 0.25 & 0.08 & 1.09 & 4.02 & 58.99 & 0.00 & 6.19 & 655.36 \\
\hline & Error & $\begin{array}{l}1 \\
8\end{array}$ & 1.28 & 2.50 & 2.50 & 5.96 & 272.15 & 160.56 & 0.24 & 0.08 & 1.31 & 5.20 & 33.10 & 2.21 & 4.87 & 150.89 \\
\hline & Total & $\begin{array}{l}2 \\
9\end{array}$ & 6.99 & 5.44 & 5.44 & 17.97 & 414.32 & 215.17 & 5.44 & 0.23 & 2.24 & 30.67 & 398.99 & 38.48 & 193.06 & 792.32 \\
\hline \multirow{7}{*}{$\begin{array}{l}\mathbf{T} \\
2\end{array}$} & Replicates & 2 & 0.43 & $7.23^{*}$ & 2.13 & 5.31 & 348.10 & 104.67 & 0.07 & 0.03 & 2.00 & $23.45^{*}$ & 75.70 & $13.06^{*}$ & 5.16 & 17.30 \\
\hline & Treatments & 9 & $19.85^{* *}$ & $9.05 * *$ & $12.05 * *$ & $44.82 * *$ & 713.36* & 349.16 & $17.06 * *$ & $0.54 * *$ & $3.95 *$ & $83.01 * *$ & $1202.60 * *$ & $116.69 * *$ & $611.19 * *$ & $2247.40 * *$ \\
\hline & Parents & 3 & 52.30 & $18.30 * *$ & $18.30 * *$ & $90.25 * *$ & 954.00 & $268.87 *$ & $28.73 * *$ & $0.77 * *$ & 1.22 & $106.54 *$ & $238.52 * *$ & $185.71 * *$ & $974.83 * *$ & $4232.35 * *$ \\
\hline & Hybrids & 5 & 1.95 & 8.05 & $8.05^{*}$ & $21.08 *$ & 709.70 & $460.06^{*}$ & $13.42 * *$ & $0.51 * *$ & $6.22 * *$ & $84.66 * *$ & $2009.78 * *$ & $98.62 * *$ & $514.00 * *$ & $1374.83 * *$ \\
\hline & $\begin{array}{l}\text { Parent } \\
\text { Vs.Hybrids }\end{array}$ & 1 & $12.22 * *$ & $13.39 *$ & $13.39 *$ & $27.48 *$ & 9.75 & 35.56 & 0.25 & 0.08 & 1.09 & 4.02 & 58.99 & 0.00 & 6.19 & 655.36 \\
\hline & Error & $\begin{array}{l}1 \\
8\end{array}$ & 1.28 & 2.50 & 2.50 & 5.96 & 272.15 & 160.56 & 0.24 & 0.08 & 1.31 & 5.20 & 33.10 & 2.21 & 4.87 & 150.89 \\
\hline & Total & $\begin{array}{l}2 \\
9\end{array}$ & 6.99 & 5.44 & 5.44 & 17.97 & 414.32 & 215.17 & 5.44 & 0.23 & 2.24 & 30.67 & 398.99 & 38.48 & 193.06 & 792.32 \\
\hline
\end{tabular}

DF- Degree of freedom, DOF $50 \%$ T- Days to 50\% tasseling, DOF 50\% S- days to 50\% silking, ASI- Anthesis silking interval, DOF 75\% B- Days to 75\% brown husk, PH- Plant height, EH- Ear height, CL- Cob length, CD- Cob diameter, NKRPC- number of kernel row per cob, NKPR- number of kernel per row, 1000 GW- 1000 grain weight, HI- Harvest index, SP- shelling percentage, GY per plant- Grain yield per plant. 
Table.2 Observed mean squares from general combining ability (GCA) and specific combining ability (SCA) in control (C), Treatment 1 (T1) and Treatment 2 (T2) condition

\begin{tabular}{|c|c|c|c|c|c|c|c|c|c|c|c|c|c|c|c|c|}
\hline & $\begin{array}{c}\text { Source } \\
\text { of } \\
\text { variation }\end{array}$ & DF & $\begin{array}{c}\text { DOF } \\
\mathbf{5 0 \% ~ T ~} \\
\text { (Days) }\end{array}$ & $\begin{array}{c}\text { DOF } \\
\mathbf{5 0 \%} \\
\text { S } \\
\text { (Days) }\end{array}$ & $\begin{array}{c}\text { ASI } \\
\text { (Days) }\end{array}$ & $\begin{array}{c}\text { DOF } \\
75 \% \mathrm{~B} \\
\text { (Days) }\end{array}$ & $\begin{array}{c}\text { PH } \\
(\mathbf{c m})\end{array}$ & $\begin{array}{c}\text { EH } \\
(\mathbf{c m})\end{array}$ & $\begin{array}{c}\text { CL } \\
(\mathrm{cm})\end{array}$ & $\begin{array}{c}\text { CD } \\
(\mathrm{cm})\end{array}$ & NKRPC & NKPR & $\begin{array}{c}1000 \\
\text { GW(gm) }\end{array}$ & HI (\%) & SP (\%) & $\begin{array}{c}\text { GY/per } \\
\text { plant } \\
\text { (gm) }\end{array}$ \\
\hline \multirow[t]{3}{*}{ C } & GCA & 3 & $14.91 * *$ & $9.12 * *$ & $1.11 * *$ & $23.24 * *$ & $318.83 * *$ & $140.80 * *$ & $13.26 * *$ & $0.389 * *$ & $1.03 *$ & $55.58 * *$ & $3157.01 * *$ & $83.61 * *$ & $513.57 * *$ & $1473.05^{* *}$ \\
\hline & SCA & 6 & $6.43 * *$ & $4.92 * *$ & $0.36^{* *}$ & $8.43 * *$ & $256.00 * *$ & $149.10 * *$ & $2.22 * *$ & $0.08 * *$ & $0.82 * *$ & $19.48 * *$ & $297.31 * *$ & $17.25^{* *}$ & $68.29 * *$ & $328.17 * *$ \\
\hline & Error & 18 & 0.42 & 0.47 & 0.12 & 2.05 & 119.15 & 64.73 & 0.056 & 0.04 & 0.28 & 2.47 & 67.01 & 0.83 & 1.75 & 34.84 \\
\hline \multirow[t]{3}{*}{ T1 } & GCA & 3 & $8.23^{* *}$ & $2.72 * *$ & $1.11 * *$ & $3.73 * *$ & $308.42 * *$ & $109.03 * *$ & $12.26 * *$ & $0.39 * *$ & $1.68 * *$ & $50.82 * *$ & $8.24 * *$ & $2.72 * *$ & $1.11 * *$ & $73 * *$ \\
\hline & SCA & 6 & $3.63 * *$ & 0.23 & $0.36 * *$ & $15.13^{* *}$ & $216.97 * *$ & $109.14 * *$ & $2.15 * *$ & $0.96 * *$ & $1.086^{* *}$ & $17.81 * *$ & $3.63 * *$ & $0.23^{* *}$ & $0.36 * *$ & $15.14 * *$ \\
\hline & Error & 18 & 0.50 & 0.52 & 0.13 & 1.68 & 96.33 & 57.21 & 0.06 & 0.03 & 0.43 & 1.82 & 0.50 & 0.52 & 0.12 & 1.68 \\
\hline \multirow[t]{3}{*}{$\mathbf{T} 2$} & GCA & 3 & $10.75 * *$ & $6.31 * *$ & $4.76^{* *}$ & $10.71^{* *}$ & $305.08 * *$ & $117.6^{* *}$ & $12.36^{* * *}$ & $0.37 * *$ & $2.00 *$ & $49.22 * *$ & $406.43 * *$ & $83.84 * *$ & $482.50 * *$ & $1707.16^{* *}$ \\
\hline & SCA & 6 & $4.57 * *$ & $1.37 * *$ & $3.65 * *$ & $17.06^{* *}$ & $204.14 * *$ & $115.76^{* *}$ & $2.34 * *$ & $0.09 * *$ & $0.99 * *$ & $16.89 * *$ & $398.08 * *$ & $16.42 * *$ & $64.34 * *$ & $270.11 * *$ \\
\hline & Error & 18 & 0.43 & 0.44 & 0.84 & 1.99 & 90.72 & 53.50 & 0.08 & 0.03 & 0.44 & 1.73 & 11.04 & 0.73 & 1.63 & 50.30 \\
\hline
\end{tabular}

DF- Degree of freedom, DOF $50 \%$ T- Days to $50 \%$ tasseling, DOF 50\% S- days to 50\% silking, ASI- Anthesis silking interval, DOF 75\% B- Days to 75\% brown husk, PH- Plant height, EH- Ear height, CL- Cob length, CD- Cob diameter, NKRPC- number of kernel row per cob, NKPR- number of kernel per row, 1000 GW- 1000 grain weight, HI- Harvest index, SP- shelling percentage, GY per plant- Grain yield per plant. 
Table.3 Estimation of GCA effects of parents for 14 traits of maize under control and treatment 1 condition

\begin{tabular}{|c|c|c|c|c|c|c|c|c|c|c|c|c|c|c|c|}
\hline & Parent & $\begin{array}{c}\text { DOF } \\
50 \% \mathrm{~T} \\
\text { (Days) }\end{array}$ & $\begin{array}{c}\text { DOF } \\
\mathbf{5 0 \% ~ S} \\
\text { (Days) }\end{array}$ & $\begin{array}{c}\text { ASI } \\
\text { (Days) }\end{array}$ & $\begin{array}{c}\text { DOF } \\
75 \% \mathrm{~B} \\
\text { (Days) }\end{array}$ & $\begin{array}{c}\text { PH } \\
(\mathbf{c m})\end{array}$ & $\begin{array}{c}\text { EH } \\
(\mathbf{c m})\end{array}$ & $\begin{array}{c}\text { CL } \\
(\mathbf{c m})\end{array}$ & $\begin{array}{c}\text { CD } \\
(\mathbf{c m})\end{array}$ & $\begin{array}{c}\text { NKRP } \\
\text { C }\end{array}$ & NKPR & $\begin{array}{c}1000 \\
\text { GW(gm) }\end{array}$ & HI (\%) & SP (\%) & $\begin{array}{c}\text { GY/per } \\
\text { plant } \\
\text { (gm) }\end{array}$ \\
\hline \multirow{6}{*}{$\begin{array}{l}\mathbf{C} \\
\mathbf{O} \\
\mathbf{N} \\
\mathbf{T} \\
\mathbf{R} \\
\mathbf{O} \\
\mathbf{L}\end{array}$} & $\mathrm{P} 1$ & $1.33 * *$ & $0.83 * *$ & $-0.50 *$ & $1.47 * *$ & 4.80 & 5.15 & $-0.25 *$ & 0.01 & 0.11 & -0.59 & -0.50 & $-1.19 * *$ & $-3.21 * *$ & $2.93 * *$ \\
\hline & $\mathrm{P} 2$ & 0.27 & 0.11 & -0.16 & 0.30 & 4.66 & 2.37 & $1.12 * *$ & $0.22 * *$ & 0.00 & $3.08 * *$ & $19.50 * *$ & $3.63 * *$ & $7.14 * *$ & $13.56^{* *}$ \\
\hline & P3 & $-0.27^{*}$ & $-1.78 * *$ & $0.50 * *$ & $-2.87 * *$ & 1.16 & -1.54 & $1.13 * *$ & 0.12 & $0.44 *$ & $1.47 * *$ & $13.06 * *$ & $2.26 * *$ & $7.72 * *$ & $10.29 * *$ \\
\hline & $\mathrm{P} 4$ & $0.66^{* *}$ & $0.83 * *$ & 0.16 & $1.08 *$ & $-10.64^{*}$ & $-5.99 *$ & $-2.00 * *$ & $-0.36 * *$ & $-0.57 * *$ & $-3.98 * *$ & $-32.06^{* *}$ & $-4.70 * *$ & $-11.66^{*}$ & $-20.93 * *$ \\
\hline & $\begin{array}{l}\mathrm{Gi} \ll 0 \\
\text { at } 95 \%\end{array}$ & $0.73 * *$ & $0.76 * *$ & $0.40 * *$ & $1.61 * *$ & $12.29 * *$ & $9.06 * *$ & $0.27 * *$ & $0.21 * *$ & $0.60 * *$ & $1.77 * *$ & $9.21 * *$ & $1.02 * *$ & $1.49 * *$ & $6.64 * *$ \\
\hline & $\begin{array}{c}\text { Gi-- } \mathrm{Gj} \text { at } \\
95 \%\end{array}$ & $1.18 * *$ & $1.25 * *$ & $0.65 * *$ & $2.63 * *$ & $20.06 * *$ & $14.78^{* *}$ & $0.43 * *$ & $0.35^{* *}$ & $0.98 * *$ & $2.88 * *$ & $15.04 * *$ & $1.68 * *$ & $2.43^{* *} *$ & $10.85^{* *}$ \\
\hline \multirow{6}{*}{$\begin{array}{l}\mathbf{T} \\
\mathbf{1}\end{array}$} & $\mathrm{P} 1$ & 0.500 & -0.083 & $-0.50 * *$ & 0.15 & 3.71 & 4.12 & $-0.32 * *$ & -0.05 & 0.11 & -0.53 & 1.00 & $-0.95 * *$ & $-3.03 * *$ & $* * 2.83$ \\
\hline & $\mathrm{P} 2$ & 0.33 & 0.41 & -0.16 & $1.05 *$ & 4.8 & 2.19 & $1.12 * *$ & $0.24 * *$ & 0.33 & $3.00 * *$ & $20.37 * *$ & $3.67 * *$ & $7.20 * *$ & $13.50 * *$ \\
\hline & P3 & $-1.72 * *$ & $-0.91 * *$ & $0.50 * *$ & $-0.64 * *$ & 2.05 & -0.74 & $1.08 * *$ & 0.11 & 0.33 & $1.31 *$ & $12.17 * *$ & $2.09 * *$ & $7.38 * *$ & $10.03 * *$ \\
\hline & $\mathrm{P} 4$ & $0.88 * *$ & $0.58 *$ & 0.16 & -0.53 & $-10.60 *$ & -5.61 & $-1.89 * *$ & $-0.34 * *$ & $-0.77 * *$ & $-3.78 * *$ & $-33.55^{* *}$ & $-4.81 * *$ & $-11.55^{*}$ & $-20.72 * *$ \\
\hline & $\begin{array}{l}\mathrm{Gi}<>0 \\
\text { at } 95 \%\end{array}$ & $0.79 * *$ & $0.80 * *$ & $0.39 * *$ & $1.45^{* *}$ & $11.04 * *$ & $8.51 * *$ & $0.26^{* *}$ & $0.19 * *$ & $0.73 * *$ & $1.51 * *$ & $8.84 * *$ & $0.98 * *$ & $1.37 * *$ & $6.33 * *$ \\
\hline & $\begin{array}{c}\text { Gi-- } \mathrm{Gj} \text { at } \\
95 \%\end{array}$ & $1.29 * *$ & $1.31 * *$ & $0.64 * *$ & $2.37 * *$ & $18.03 * *$ & $13.89 * *$ & $0.44 * *$ & $0.32 * *$ & $1.19 * *$ & $2.47 * *$ & $14.44 * *$ & $1.61 * *$ & $2.23 * *$ & $10.34 * *$ \\
\hline
\end{tabular}

DF- Degree of freedom, DOF $50 \%$ T- Days to 50\% tasseling, DOF 50\% S- days to 50\% silking, ASI- Anthesis silking interval, DOF $75 \%$ B- Days to $75 \%$ brown husk, PH- Plant height, EH- Ear height, CL- Cob length, CD- Cob diameter, NKRPC- number of kernel row per cob, NKPR- number of kernel per row, 1000 GW- 1000 grain weight, HI- Harvest index, SP- shelling percentage, GY per plant- Grain yield per plant 
Table.4 Estimation of GCA effects of parents for 14 traits of maize under treatment 2 condition

\begin{tabular}{|c|c|c|c|c|c|c|c|c|c|c|c|c|c|c|c|}
\hline & Parent & $\begin{array}{c}\text { DOF } \\
\mathbf{5 0 \%} \mathrm{T} \\
\text { (Days) }\end{array}$ & $\begin{array}{c}\text { DOF } \\
50 \% \mathrm{~S} \\
\text { (Days) }\end{array}$ & $\begin{array}{c}\text { ASI } \\
\text { (Days) }\end{array}$ & $\begin{array}{c}\text { DOF } \\
75 \% \mathrm{~B} \\
\text { (Days) }\end{array}$ & $\begin{array}{c}\text { PH } \\
(\mathrm{cm})\end{array}$ & $\begin{array}{c}\text { EH } \\
(\mathrm{cm})\end{array}$ & $\begin{array}{c}\text { CL } \\
(\mathrm{cm})\end{array}$ & CD (cm) & NKRPC & NKPR & $\begin{array}{c}1000 \\
\text { GW(gm) }\end{array}$ & HI (\%) & SP (\%) & $\begin{array}{c}\text { GY/per } \\
\text { plant } \\
\text { (gm) }\end{array}$ \\
\hline \multirow[t]{6}{*}{ T2 } & P1 & 0.36 & $1.16^{* *}$ & 0.38 & 0.64 & 3.58 & 4.31 & $-0.27 *$ & 0.03 & -0.16 & -0.52 & -1.88 & $-0.98 *$ & $-2.79 * *$ & 1.94 \\
\hline & $\mathrm{P} 2$ & $0.63 *$ & $-0.55^{*}$ & $-1.05^{* *}$ & 0.76 & 5.41 & 2.52 & $1.15^{* *}$ & $0.23 * *$ & 0.27 & $2.93 *$ & $5.05^{* *}$ & $3.68 * *$ & $7.13 * *$ & $14.24 * *$ \\
\hline & P3 & $-1.97 *$ & $-1.11 *$ & $1.00 * *$ & $-2.00 *$ & 1.41 & -1.12 & $1.04 * *$ & 0.10 & 0.61 & $1.32 *$ & $7.61 * *$ & $2.10^{* *}$ & $7.13 * *$ & $10.75^{* *}$ \\
\hline & $\mathrm{P} 4$ & $0.97 * *$ & $0.50 *$ & -0.33 & 0.59 & $-10.41 *$ & $-5.79 *$ & $-1.92 * *$ & $-0.34 *$ & -0.72 & $-3.73^{*}$ & $-10.77 *$ & $-4.79 *$ & $-11.4 * *$ & $-23.05^{*}$ \\
\hline & $\begin{array}{c}\mathrm{Gi} \ll 0 \text { at } \\
95 \%\end{array}$ & $0.73 * *$ & $0.74 * *$ & $1.02 * *$ & $1.58 * *$ & $10.71 * *$ & $8.23 * *$ & $0.31 * *$ & $0.19 * *$ & 0.74 & $1.48^{*}$ & $3.73 * *$ & $0.96 * *$ & $1.43 * *$ & $7.90 * *$ \\
\hline & $\begin{array}{c}\mathrm{Gi}-\mathrm{-Gj} \text { at } \\
95 \%\end{array}$ & $1.20 * *$ & $1.21 * *$ & $1.67 * *$ & $2.59 * *$ & $17.50 * *$ & $13.43 * *$ & $0.52 * *$ & $0.31 * *$ & 1.21 & $2.42 *$ & $6.10 * *$ & $1.57 * *$ & $2.34 * *$ & $13.03 * *$ \\
\hline
\end{tabular}

DF- Degree of freedom, DOF $50 \%$ T- Days to $50 \%$ tasseling, DOF 50\% S- days to 50\% silking, ASI- Anthesis silking interval, DOF 75\% B- Days to 75\% brown husk, PH- Plant height, EH- Ear height, CL- Cob length, CD- Cob diameter, NKRPC- number of kernel row per cob, NKPR- number of kernel per row, 1000 GW- 1000 grain weight, HI- Harvest index, SP- shelling percentage, GY per plant- Grain yield per plant 
Table.5 Estimation of SCA effect of $F_{1}$ hybrids for 14 traits of maize under control

\begin{tabular}{|c|c|c|c|c|c|c|c|c|c|c|c|c|c|c|c|}
\hline & Hybrid & $\begin{array}{c}\text { DOF } \\
\mathbf{5 0 \%} \text { T } \\
\text { (Days) }\end{array}$ & $\begin{array}{c}\text { DOF } \\
\mathbf{5 0 \% ~ S} \\
\text { (Days) }\end{array}$ & $\begin{array}{c}\text { ASI } \\
\text { (Days) }\end{array}$ & $\begin{array}{c}\text { DOF } \\
75 \% \mathrm{~B} \\
\text { (Days) }\end{array}$ & $\begin{array}{c}\text { PH } \\
(\mathbf{c m})\end{array}$ & $\begin{array}{c}\text { EH } \\
(\mathbf{c m})\end{array}$ & $\begin{array}{c}\text { CL } \\
(\mathrm{cm})\end{array}$ & $\begin{array}{c}\text { CD } \\
(\mathbf{c m})\end{array}$ & NKRPC & NKPR & $\begin{array}{c}1000 \\
\text { GW(gm) }\end{array}$ & $\begin{array}{l}\text { HI } \\
(\%)\end{array}$ & $\operatorname{SP}(\%)$ & $\begin{array}{c}\text { GY/per } \\
\text { plant } \\
\text { (gm) }\end{array}$ \\
\hline \multirow[t]{9}{*}{ c } & P1 X P2 & $-1.67 *$ & -0.94 & 0.73 & -0.81 & -6.76 & -8.51 & $1.61^{* *}$ & -0.01 & 0.56 & $5.80 * *$ & 8.80 & $5.19 * *$ & $10.06 * *$ & $12.07 * *$ \\
\hline & P1 XP3 & 0.21 & 0.27 & 0.06 & 0.69 & -3.26 & -3.71 & $1.73 * *$ & $0.51 *$ & 0.11 & 3.078 & $23.57 *$ & $4.01 * *$ & $7.95 * *$ & 8.38 \\
\hline & P1 X P4 & $-1.73 *$ & $-2.00 *$ & -0.26 & -1.92 & $23.60 *$ & 16.52 & -0.11 & -0.13 & -0.22 & -0.47 & 0.02 & -0.39 & -1.87 & -2.61 \\
\hline & P2 X P3 & $3.26 * *$ & $3.00 * *$ & -0.26 & $3.52 *$ & -2.23 & 2.350 & $\begin{array}{c}- \\
1.93 * *\end{array}$ & -0.29 & 0.88 & $-3.99 *$ & -11.08 & $-3.80 *$ & $-6.17 * *$ & $23.49 * *$ \\
\hline & P2 X P4 & -1.01 & -0.27 & 0.73 & -1.42 & 12.07 & 5.96 & $\begin{array}{c}- \\
0.82^{*} *\end{array}$ & -0.15 & $-1.44^{*}$ & $-4.14^{*}$ & -1.31 & $-3.76^{*}$ & $-9.89 * *$ & $\begin{array}{c}- \\
24.06^{* *}\end{array}$ \\
\hline & P3 X P4 & $2.87 * *$ & $2.27 * *$ & -0.60 & $3.74^{*}$ & -22.09 & $\begin{array}{c}- \\
19.45^{*}\end{array}$ & -0.11 & -0.07 & -0.55 & -2.53 & $-23.86^{*}$ & -1.57 & 1.963 & 0.727 \\
\hline & $\begin{array}{l}\mathrm{Sij} \Leftrightarrow 0 \\
\text { at } 95 \%\end{array}$ & 1.42 & 1.50 & 0.77 & 3.15 & 24.02 & 17.71 & 0.51 & 0.41 & 1.16 & 3.45 & 18.02 & 2.07 & 2.91 & 12.99 \\
\hline & $\begin{array}{l}\text { Sij--Sik } \\
\text { at } 95 \%\end{array}$ & 2.141 & 2.264 & 1.166 & 4.752 & 36.225 & 26.700 & 0.775 & 0.627 & 1.761 & 5.211 & 27.166 & 3.026 & 4.387 & 19.588 \\
\hline & $\begin{array}{l}\text { Sij--Skl } \\
\text { at } 95 \%\end{array}$ & 1.915 & 2.025 & 1.043 & 4.251 & 32.401 & 23.881 & 0.693 & 0.561 & 1.575 & 4.661 & 24.298 & 2.707 & 3.924 & 17.520 \\
\hline
\end{tabular}

DF- Degree of freedom, DOF $50 \%$ T- Days to $50 \%$ tasseling, DOF 50\% S- days to 50\% silking, ASI- Anthesis silking interval, DOF $75 \%$ B- Days to $75 \%$ brown husk, PH- Plant height, EH- Ear height, CL- Cob length, CD- Cob diameter, NKRPC- number of kernel row per cob, NKPR- number of kernel per row, 1000 GW- 1000 grain weight, HI- Harvest index, SP- shelling percentage, GY per plant- Grain yield per plant 
Table.6 Estimation of SCA effect of $\mathrm{F}_{1}$ hybrids for 14 traits of maize under T1

\begin{tabular}{|c|c|c|c|c|c|c|c|c|c|c|c|c|c|c|c|}
\hline & Hybrid & $\begin{array}{c}\text { DOF } \\
50 \% \mathrm{~T} \\
\text { (Days) }\end{array}$ & $\begin{array}{c}\text { DOF } \\
50 \% \mathrm{~S} \\
\text { (Days) }\end{array}$ & $\begin{array}{c}\text { ASI } \\
\text { (Days) }\end{array}$ & $\begin{array}{c}\text { DOF } \\
75 \% \mathrm{~B} \\
\text { (Days) }\end{array}$ & $\begin{array}{c}\text { PH } \\
(\mathrm{cm})\end{array}$ & $\begin{array}{c}\text { EH } \\
(\mathrm{cm})\end{array}$ & $\begin{array}{c}\text { CL } \\
(\mathrm{cm})\end{array}$ & $\begin{array}{c}\text { CD } \\
(\mathrm{cm})\end{array}$ & NKRPC & NKPR & $\begin{array}{c}1000 \\
\text { GW(gm) }\end{array}$ & $\begin{array}{l}\text { HI } \\
(\%)\end{array}$ & SP $(\%)$ & $\begin{array}{c}\text { GY/per } \\
\text { plant } \\
\text { (gm) }\end{array}$ \\
\hline \multirow[t]{9}{*}{ T1 } & P1 X P2 & -1.36 & -0.43 & 0.73 & 1.25 & -4.86 & -7.16 & $1.72 * *$ & 0.03 & $1.56^{*}$ & $5.54 * *$ & 7.78 & $4.81 * *$ & $9.50 * *$ & $11.59 * *$ \\
\hline & P1 XP3 & $1.68^{*}$ & 0.23 & 0.06 & -0.11 & -1.98 & -3.42 & $1.64 * *$ & $0.53^{*}$ & 0.22 & $3.17 *$ & $24.10^{*}$ & $4.00 * *$ & $7.59 * *$ & 8.29 \\
\hline & P1 X P4 & -1.25 & -0.60 & -0.26 & $-3.19 *$ & $21.29 *$ & 14.81 & -0.11 & -0.13 & 0.00 & -0.56 & 0.67 & -0.21 & -1.30 & -2.10 \\
\hline & P2 X P3 & $2.18^{*}$ & 0.06 & -0.26 & $3.23 *$ & -2.95 & 1.48 & $-1.81 * *$ & -0.39 & 0.667 & $-3.85^{*}$ & -11.81 & $-3.74 *$ & $-5.99 * *$ & $22.77 * *$ \\
\hline & P2 X P4 & -0.42 & -0.43 & 0.73 & $-4.28 * *$ & 10.260 & 4.795 & $-0.89 * *$ & -0.17 & -0.88 & $-3.88^{*}$ & -2.35 & $-3.63 *$ & $-9.46 * *$ & $-23.23 *$ \\
\hline & P3 X P4 & $1.63^{*}$ & 0.23 & -0.60 & $5.44 * *$ & $-21.32 *$ & $-16.07 *$ & -0.06 & -0.06 & -0.88 & -2.21 & $-21.93 *$ & -1.43 & 1.43 & -0.44 \\
\hline & $\begin{array}{l}\text { Sij } \Leftrightarrow 0 \\
\text { at } 95 \%\end{array}$ & 1.555 & 1.579 & 0.773 & 2.850 & 21.605 & 16.650 & 0.527 & 0.385 & 1.435 & 2.968 & 17.309 & 1.931 & 2.680 & 12.393 \\
\hline & $\begin{array}{l}\text { Sij--Sik } \\
\text { at } 95 \%\end{array}$ & 2.344 & 2.381 & 1.166 & 4.296 & 32.571 & 25.101 & 0.795 & 0.580 & 2.163 & 4.474 & 26.094 & 2.912 & 4.041 & 18.684 \\
\hline & $\begin{array}{l}\text { Sij--Skl } \\
\text { at } 95 \%\end{array}$ & 2.096 & 2.130 & 1.043 & 3.843 & 29.132 & 22.451 & 0.711 & 0.519 & 1.934 & 4.002 & 23.339 & 2.604 & 3.614 & 16.711 \\
\hline
\end{tabular}

DF- Degree of freedom, DOF $50 \%$ T- Days to $50 \%$ tasseling, DOF 50\% S- days to 50\% silking, ASI- Anthesis silking interval, DOF 75\% B- Days to 75\% brown husk, PH- Plant height, EH- Ear height, CL- Cob length, CD- Cob diameter, NKRPC- number of kernel row per cob, NKPR- number of kernel per row, 1000 GW- 1000 grain weight, HI- Harvest index, SP- shelling percentage, GY per plant- Grain yield per plant 
Table.7 Estimation of SCA effect of $\mathrm{F}_{1}$ hybrids for 14 traits of maize under $\mathrm{T} 2$

\begin{tabular}{|c|c|c|c|c|c|c|c|c|c|c|c|c|c|c|c|}
\hline & Hybrid & $\begin{array}{c}\text { DOF } \\
\mathbf{5 0 \%} \mathbf{T} \\
\text { (Days) }\end{array}$ & $\begin{array}{c}\text { DOF } \\
\mathbf{5 0 \% ~ S} \\
\text { (Days) }\end{array}$ & $\begin{array}{c}\text { ASI } \\
\text { (Days) }\end{array}$ & $\begin{array}{c}\text { DOF } \\
75 \% \text { B } \\
\text { (Days) }\end{array}$ & $\begin{array}{c}\text { PH } \\
(\mathrm{cm})\end{array}$ & $\begin{array}{c}\text { EH } \\
(\mathrm{cm})\end{array}$ & $\begin{array}{c}\text { CL } \\
(\mathrm{cm})\end{array}$ & $\begin{array}{c}\text { CD } \\
(\mathbf{c m})\end{array}$ & NKRPC & NKPR & $\begin{array}{c}1000 \\
\text { GW(gm) }\end{array}$ & $\begin{array}{l}\text { HI } \\
(\%)\end{array}$ & SP (\%) & $\begin{array}{c}\text { GY/per } \\
\text { plant } \\
(\text { gm) }\end{array}$ \\
\hline \multirow[t]{9}{*}{ c } & P1 X P2 & -1.03 & -0.74 & 0.73 & 0.69 & -5.04 & -6.74 & $1.67 * *$ & 0.03 & 0.48 & $5.40 * *$ & $15.70^{* *}$ & $4.96 * *$ & $9.59 * *$ & $10.83 * *$ \\
\hline & P1 XP3 & $1.57 *$ & $1.47 *$ & 0.34 & 0.91 & -2.22 & -3.36 & $1.83 * *$ & $0.51 *$ & 0.15 & $3.04 *$ & $15.47 * *$ & $4.02 * *$ & $7.89 * *$ & 7.62 \\
\hline & P1 X P4 & $-1.70 *$ & $-1.80 *$ & 0.34 & -2.87 & $20.45^{*}$ & 14.95 & -0.13 & -0.13 & 0.15 & -0.52 & $-21.13 * *$ & -.0 .16 & -1.42 & 0.40 \\
\hline & P2 X P3 & $1.96^{*}$ & -0.80 & $-2.87 *$ & $4.81 * *$ & -0.35 & 1.98 & $\begin{array}{c}- \\
1.93 * *\end{array}$ & -0.30 & $1.71 *$ & $-3.70 *$ & 6.53 & $-3.73 *$ & $-6.05 * *$ & $23.15^{* *}$ \\
\hline & P2 X P4 & -0.31 & 0.25 & 0.45 & $-3.82 *$ & 10.92 & 4.93 & $-0.86^{*}$ & -0.16 & -0.95 & $-3.80 *$ & $-24.41 * *$ & $-3.69 *$ & $-9.57 * *$ & $-20.46 *$ \\
\hline & P3 X P4 & $2.63 * *$ & 0.47 & $-2.26^{*}$ & $4.94 * *$ & $-20.96^{*}$ & $-17.10^{*}$ & -0.10 & -0.06 & -0.62 & -2.20 & $14.70 * *$ & -1.39 & 1.80 & 1.85 \\
\hline & $\begin{array}{l}\mathrm{Sij} \ll 0 \\
\text { at } 95 \%\end{array}$ & 1.441 & 1.453 & 2.011 & 3.103 & 20.967 & 16.102 & 0.623 & 0.376 & 1.459 & 2.899 & 7.313 & 1.891 & 2.807 & 15.612 \\
\hline & $\begin{array}{l}\text { Sij--Sik } \\
\text { at } 95 \%\end{array}$ & 2.172 & 2.191 & 3.032 & 4.678 & 31.609 & 24.274 & 0.940 & 0.567 & 2.200 & 4.370 & 11.025 & 2.850 & 4.232 & 23.536 \\
\hline & $\begin{array}{l}\text { Sij--Skl } \\
\text { at } 95 \%\end{array}$ & 1.943 & 1.960 & 2.712 & 4.184 & 28.272 & 21.711 & 0.841 & 0.507 & 1.968 & 3.909 & 9.861 & 2.549 & 3.785 & 21.052 \\
\hline
\end{tabular}

DF- Degree of freedom, DOF $50 \%$ T- Days to 50\% tasseling, DOF 50\% S- days to 50\% silking, ASI- Anthesis silking interval, DOF 75\% B- Days to 75\% brown husk, PH- Plant height, EH- Ear height, CL- Cob length, CD- Cob diameter, NKRPC- number of kernel row per cob, NKPR- number of kernel per row, 1000 GW- 1000 grain weight, HI- Harvest index, SP- shelling percentage, GY per plant- Grain yield per plant 


\section{Test weight}

SCA effect for test weight under control only one cross P1 X P3 (23.57) observed significant positive whereas under treatment 1 cross P1 X P3 (24.10) was again found to be significant positive. Under treatment 2, crosses P1 X P2 (15.70), P1 X P3 (15.47) and P3 X P4 (14.70) were found be highly significant positive in desirable direction (Table.6).

\section{Harvest index}

SCA effect for harvest index under control for crosses P1 X P2 (5.19\%) and P1 X P3 $(4.01 \%)$ were highly significant positive in desirable direction. Under treatment 1 , crosses P1 X P2 (4.81\%) and P1 X P3 (4.0\%) were also highly significant positive whereas under treatment 2, again crosses P1 X P2 (4.96\%) and P1 X P3 (4.02\%) were also highly significant positive in desirable direction.

\section{Shelling percentage}

SCA effect for shelling percentage under control for crosses P1 X P2 (10.06\%) and P1 X P3 (7.95\%) were highly significant positive in desirable direction. Under treatment 1 , crosses P1 X P2 (9.50\%) and P1 X P3 (7.59\%) were also highly significant positive whereas under treatment 2, again crosses P1 X P2 (9.59\%) and P1 X P3 (7.89\%) were also highly significant positive in desirable direction (Table.7).

\section{Grain yield per plant}

SCA effect for grain yield per plant under control for crosses P1 X P2 (12.07 gm) and P1 X P3 (8.38 gm) were highly significant positive in desirable direction. Under treatment 1, crosses P1 X P2 (11.59 gm) and P1 $\quad$ X $\quad$ P3 $\quad(8.29$ gm) were also highly significant positive whereas under treatment
2, again crosses P1 X P2 (10.83 gm) and P1 X P3 (7.62 gm) were also highly significant positive in desirable direction.

The effect of SCA and GCA effect of parents exhibited significant interaction for all characters indicating that there was presence of exploitable variation among parents in terms of heterosis. Term specific combining ability and general combining ability was originally defined by Sprague and Tatum in 1942. General combining ability was average performance of a line in all combinations while SCA was specific for a particular cross. General combining ability gives estimates of average performance of parents in all the crosses thus enabling the parents to have effective participation in crosses for producing superior $\mathrm{F} 1$ hybrids in all combinations. In the results obtained by this investigation parents $\mathrm{P} 3$ was found to have significant GCA for 11 characters, P2 for 9 characters, $\mathrm{P} 4$ for two characters while P1 for one character. Specific combining ability is assessed for the particular crosses leading to identification of best pairs for F1 production. The crosses performing best were $\mathrm{P} 1 \mathrm{xP} 4$ for three characters, P2X P3 for 2 characters, P3X P4 for 4 characters, P2 X P4 for one character, P1X P3 for 7 characters and P1 X $\mathrm{P} 2$ for 6 characters. The mean squares for general and specific combining abilities for characters indicated significant differences among the GCA as well as SCA effects. This suggested presence of notable genetic variability among the genotypes for the traits taken for this study. Narro et al., (2003) reported highly significant differences for most of the sources of variation. The significant differences for gca and sca variances for different traits in maize have been reported earlier (Mathur and Bhatnagar, 1995). The mean squares of genotypes (diallel hybrids) were highly significant for all the traits. There was presence of sufficient amount of variability for these studies traits. 
Further, analysis of variance for combining ability showed that estimates of mean squares due to GCA and SCA were highly significant for all the characters. This exhibited importance of both additive and non-additive components of genetic variance in controlling these traits. This was confirmed by Debnath and Sarker (1990) and Derera et al., (2007) who reported similar results for yield and yield components in maize. Importance of both additive and nonadditive gene effects in maize were also reported by Rokadia and Kaushik (2005).

\section{References}

Rokadia P, Kaushik SK. Exploitation of combining ability for heterosis in maize (Zea mays L.). In: Pixley K, Zhang SH. (ed). Proc. 9th Asian Reg. Maize Workshop. Beijing, China. 2005, 89-91.

Derera P, Tongoona BS, Vivek N, Van RP, Laing MD (2017) Gene action determining Phaeosphaeria leaf spot disease resistance in experimental maize hybrids J. ,South Africa Journal of Plant Soil 24(3): 101-104.

Debnath, S.C. and K.R. Sarker. 1990. Combining ability analysis of grain yield and some of its attributes in maize (Zea mays L.). Indian Journal Genet. 50: 57-61.

Mathur RK, Bhatnagar SK. 1995. Partial diallel cross analysis for grain yield and its component characters in maize ( $\mathrm{Zea}$ mays L.). Annals of Agricultural Research 16(3), 324-329.

Narro, Luis; Pandey, Shivaji; Crossa, José; De León, Carlos and Salazar, Fredy. (2003). Using Line $\times$ Tester Interaction for the Formation of Yellow Maize Synthetics Tolerant to Acid Soils. Crop Science - CROP SCIENCE 43. 10.2135/cropsci2003.1718.

$\mathrm{Ke}-\mathrm{Cl}$ Xiao, Lin - Liu Hua, Bo- Hao Yu, Wang - ZHANG Ji, Peng LIU and Shu - ting DONG (2012). Arsenic Distribution Species and its Effect on maize Growth treated with Arsenate. Journal of Integrative Agriculture. 11(3): 416-423.

Sharples J M, Meharg A A, Chambers S M, Cairney J W G. 2000. Evolution: Symbiotic solution to arsenic contamination. Nature, 404, 951-952.

$\mathrm{Ke}$ - Cl Xiao, Lin - Liu Hua, Bo- Hao Yu, Wang - ZHANG Ji, Peng LIU and Shu - ting DONG (2012). Arsenic Distribution Species and its Effect on maize Growth treated with Arsenate. Journal of Integrative Agriculture. 11(3): 416-423.

Talukder MZA, Karim ANMS, Ahmed S, Amiruzzaman M (2016) Combining Ability And Heterosis On Yield And Its Component Traits In Maize (Zea mays L.). Bangladesh Journal of Agriculture Research 41(3): 565-577.

\section{How to cite this article:}

Girish Nandan, Shyam Sundar Mandal, Dr. Bishnu Deo Prasad, Urusha Zareen and Jyoti Rani. 2020. Combining Ability for Yield and its Attributes in Response to Arsenic Absorption in Maize (Zea mays L.). Int.J.Curr.Microbiol.App.Sci. 9(06): 1007-1021. doi: https://doi.org/10.20546/ijcmas.2020.906.126 